\title{
Power indices expressed in terms of minimal winning coalitions*
}

\author{
Fabien Lange ${ }^{\dagger} \quad$ László Á. Kóczy ${ }^{\ddagger}$
}

\begin{abstract}
A voting situation is given by a set of voters and the rules of legislation that determine minimal requirements for a group of voters to pass a motion. A priori measures of voting power, such as the Shapley-Shubik index and the Banzhaf value, show the influence of the individual players in a voting situation and are calculated by looking at marginal contributions in a simple game consisting of winning and losing coalitions derived from the legislative rules.

We introduce a new way to calculate these measures directly from the set of minimal winning coalitions and derive explicit formulae for the Shapley-Shubik and Banzhaf values. This new approach logically appealing as it writes measures as functions of the rules of the legislation. For certain classes of games that arise naturally in applications the logical shortcut drastically simplifies the numerical calculations to obtain the indices. The technique generalises directly to all semivalues.

Keywords. Shapley-Shubik index, Banzhaf index, semivalue, minimal winning coalition, Möbius transform.

\section{JEL codes: C71, D72}

*The authors thank the funding of the OTKA (Hungarian Fund for Scientific Research) for the project "The Strong the Weak and the Cunning: Power and Strategy in Voting Games" (NF-72610) and the second author also of the Hungarian Academy of Sciences under the Momentum Programme (LP-004/2010.

${ }^{\dagger}$ GREThA, Université Montesquieu Bordeaux $4 . \quad$ Email: fabien. lange@u-bordeaux4.fr

${ }^{\ddagger}$ Centre for Economic and Regional Studies, Hungarian Academy of Sciences, Budaörsi 45, H-1112 Budapest and Óbuda University, Budapest. Email: koczy@iehas.hu
\end{abstract}

The final publication is available at Springer via http://dx.doi.org/10.1007/s00355-012-0685-z 


\section{Introduction}

The distribution of power among voting parties remains the central question of the study of voting situations. Here power corresponds to the ability to change decisions by being a critical or swing voter to one or more coalitions. Power measures describe this ability by real numbers. While there are at least a handful of competing measures, the Shapley-Shubik and BanzhafPenrose measures are probably the most popular of all: Shapley and Shubik (1954) consider all possible orderings of voters and look at the proportion of swing voters among them, whereas Penrose (1946) and Banzhaf (1965) simply observe for each voter the number of times he is pivotal in the game. We study the calculation of these measures.

The Shapley-Shubik and Banzhaf-Penrose measures can both be expressed in terms of the marginal contributions of the players, weighted according to coefficients that depend on the measure not on the particular game at hand. This feature is shared by other semivalues, that is, values characterised by the transfer, anonymity and null player axioms, but not by all measures. The Deegan-Packel (Deegan and Packel, 1978) and public good (Holler and Packel, 1983) indices are notable exceptions.

In the context of cooperative game theory, Harsányi (1963) considers for any $n$-person game a set of bargaining subgames played by the members of each possible subset. The Harsányi dividends correspond to a kind of discrete derivative of the game and are expressing the pure contributions of cooperation (Billot and Thisse, 2005) in the given subset. This leads to another representation of the game: its Möbius transform (See Grabisch et al., 2000, and others).

A voting situation can be given by an explicit list of coalitions that can make decisions. Often, however, not all coalitions are listed, but only the rules that must be met for a coalition to be winning. For instance, the European Union has complex rules regarding the number of members and their total population to decide whether a coalition of countries is winning in the European Council (Kóczy, 2011). When calculating power indices, the common approach is to generate the corresponding (simple) cooperative game, apply the values to this game and hence obtain the results. We consider this an unnecessary detour that can be eliminated and this shortcut can, in some cases drastically reduce the required calculations.

We establish a strong link between minimal winning coalitions of a voting game, and its Möbius transform. By expressing any voting game as an alternating sum with a length corresponding to the cardinality of the set of minimal winning coalitions, we obtain a new representation of semivalues 
that is based on minimal winning coalitions only. Indeed, we want to stress that although the set of winning coalitions determines the set of minimal winning coalitions and conversely, only the minimal winning coalitions should come into play in the calculation of acceptable indices, as it it is the case for the Deegan-Packel and Holler-Packel indices.

The paper is organized as follows. In Section 2, the basic notions about voting games are briefly reviewed, and the concept of semivalue is introduced. We also recall the Shapley-Shubik and Banzhaf indices. In Section 3, we explain the Möbius transform of a game, presenting both the theory and the intuition. We present our contribution, that is, the direct formulae using the sole information of the set of minimal winning coalitions in Section 4. It is shown that such a (nonnormalized) expression characterizes the class of semivalues. In Section 5 we give several applications, and show the effectiveness of our formulae in these contexts. Finally, some concluding remarks, including literary comparisons are made in Section 6.

\section{Voting games and power indices}

The purpose of this section is to introduce the notation and primitives of our setup. We begin by clarifying some mathematical notation to avoid confusion although these will be familiar to most readers. Then we introduce games, voting games, power and power measures. There exists a plethora of power measures: we can tell which of these is more fit for a particular problem by looking at their properties. At the end we present a well-known characterisation for the two best known measures.

Let $\mathbb{N}$ denote the set of positive integers, $\mathbb{R}$ the real numbers, and $\mathbb{R}_{+}$the non negative numbers in $\mathbb{R}$. If no ambiguity occurs, the cardinality of sets $S, T, \ldots$ is denoted by lower case letters $s, t, \ldots$, and we omit curly brackets for singletons, so we write $i$ for $\{i\}$.

We denote the set of players by $N=\{1, \ldots, n\}$ and the set of possible coalitions of players by $2^{N}$. A cooperative transferable utility (TU) game over $N$ is any function $v: 2^{N} \rightarrow \mathbb{R}$ such that $v(\emptyset)=0$, where $v(S)$ represents the collective payoff that the players in coalition $S$ can achieve if it forms.

We are interested in voting games that are simple, monotone and proper. Simple games can only take the values 1 and 0 corresponding to winning and losing coalitions. In monotone games additional players can only add to the value of the coalition, that is $v(S) \leq v(T)$ for every coalition $S \subseteq T$ and proper. Finally we assume that the assembly cannot make two or more decisions simultaneously: $v(S)+v(N \backslash S) \leq 1$. Let $\mathcal{G}_{V}(N)$ denote the set of voting games on $N$. In voting games it is common to refer to players as 
voters so we use the terms interchangeably.

A voter $i$ is a swing for a coalition $S$ if $S \cup i$ is winning but $S$ is not. A winning coalition $S$ is minimal if every voter $i \in S$ is a swing for $S \backslash i$. Let $\mathcal{W}(v)$ and $\mathcal{M}(v)$ respectively denote the set of winning and minimal winning coalitions of $v$. We drop the reference to $v$ where no ambiguity occurs.

Games with $|\mathcal{M}(v)|=1$ are called unanimity games. In the unanimity game $u_{S}$ the agreement of the members of $S$ on an issue is necessary and sufficient to pass a proposal. Unanimity games are used also beyond the voting power literature.

A weighted voting game is a voting game entirely determined by a vector $\left[q ; w_{1}, \ldots, w_{n}\right]$, where $w_{i} \in \mathbb{N}$ is the weight of the voter $i$, and $q>\frac{1}{2} \sum_{i=1}^{n} w_{i}$ is the quota. This quota is the condensed form of the rules of the legislation. When the total weights of a coalition reach this threshold, it can pass a bill, that is, $S$ is winning if and only if $\sum_{i \in S} w_{i} \geq q$. For instance, in an assembly of parties, the majority rule states that a coalition is winning if the sum of the votes (weights) of all members of the coalition is greater than the half of the total of weights.

A measure of voting power is any mapping $\phi: \mathcal{G}_{V}(N) \rightarrow \mathbb{R}_{+}^{N}$, where $\phi_{i}(v)$ is a measure of the voter $i$ 's influence in the game $v$. The bigger the value $\phi_{i}(v)$, the more significant is the intrinsic power of representation of voter $i$ in game $v$. For the moment we leave the word significance ambiguous, all we can say now is that its meaning is linked with the choice of $\phi$, or rather its properties. A power measure is a power index if the $\phi_{i}(v)$ 's add up to 1 .

Such solution concepts are not limited to voting games - we can find their more general counterparts in non-simple TU games that are neither proper nor monotone. In that context, the term is value, and values are employed to find the fair allocation of gains among participants. For instance, the value Sh due to Shapley (1953) assigns to each player his average marginal contribution when forming the grand coalition in a random order, whereas the Banzhaf value Bz is simply each player's average contribution to the worths of embedding coalitions. Mathematically, for any TU-game $v$, and any player $i \in N$, we have

$$
\begin{aligned}
\mathrm{Sh}_{i}(v) & =\sum_{S \subseteq N: i \in S} \frac{(s-1) !(n-s) !}{n !}(v(S)-v(S \backslash i)), \\
\mathrm{Bz}_{i}(v) & =\frac{\sum_{S \subseteq N: i \in S}(v(S)-v(S \backslash i))}{2^{n-1}} .
\end{aligned}
$$

Applying the first to a voting game $v$ Shapley and Shubik (1954) define the Shapley-Shubik index of voter $i$ as the probability that $i$ is a swing in a given 
increasing sequence $\left(\emptyset,\left\{i_{1}\right\},\left\{i_{1}, i_{2}\right\}, \ldots, N\right)$, in other words the probability that the winning coalitions of the sequence are precisely those containing $i$.

Now let $\eta_{i}(v)$ be the number of coalitions for which $i$ is a swing in $v$. Banzhaf (1965) defined the Banzhaf index (also called Banzhaf-Coleman in$\operatorname{dex}) \beta_{i}(v)$ as the ratio of swings of $i$ over the total number of swings.

In both cases, it follows that the Shapley-Shubik index and the Banzhaf power of measure are also mathematically given by (1) and (2). Moreover, for a given game $v \in \mathcal{G}_{V}(N)$, the Banzhaf index $\beta(v)$ is proportional to $\mathrm{Bz}(v)$.

$$
\beta_{i}(v)=\frac{\eta_{i}(v)}{\sum_{j=1}^{n} \eta_{j}(v)} .
$$

Having several solutions to a problem is just as bad as having none: Which of these measures is the most appropriate? The fundamental idea to study full characterisations is to translate the above complex mathematical formulae into elementary properties and if these properties or axioms uniquely determine a measure, selecting a measure is equivalent to shopping among the axioms. The characterisations of the Shaple-Shubik index and the Banzhaf measure turn out to be rather similar. In the following we briefly recall below the well-known axioms.

Null player axiom (Null): for all games $v \in \mathcal{G}_{V}(N)$, if $i \in N$ is a null player, i.e., $i$ is a swing for no coalition $S \subseteq N \backslash i$, then $\phi_{i}(v)=0$.

Anonymity axiom (Anon): for all games $v \in \mathcal{G}_{V}(N)$, for all permutation $\sigma$ on $N$, and any $i \in N, \phi_{\sigma(i)}(v)=\phi_{i}(v \circ \sigma)$,

where $v \circ \sigma(S)$ is defined to be $v(\{\sigma(i): i \in S\})$. In words, the power of the voters should not depend on their labelling.

Efficiency (Eff): for all games $v \in \mathcal{G}_{V}(N), \sum_{i=1}^{n} \phi_{i}(v)=1$.

The following property applies to general TU-games, it does not really make sense for voting games. Nevertheless, we present it, due to its utility in the sequel.

Linearity (Lin): for all TU-games $v$ and $w$, and scalars $a, b$, then $\phi(a \cdot v+b \cdot w)=a \phi(v)+b \phi(w)$, 
where operations + and $\cdot$ of the first member denote the usual sum and scalar product in the vector space of TU-games.

Dubey (1975) overcomes the problem by introducing the transfer axiom, revisited by Laruelle and Valenciano (2001) in the following intuitive form:

Transfer axiom (Trans): for all $v, w \in \mathcal{G}_{V}(N), S \in \mathcal{M}(v) \cap$ $\mathcal{M}(w)$, and $i \in N$, then

$$
\phi_{i}(v)-\phi_{i}\left(v_{\backslash S}\right)=\phi_{i}(w)-\phi_{i}\left(w_{\backslash S}\right),
$$

where $v_{\backslash S}$ is defined by $\mathcal{W}\left(v_{\backslash S}\right):=\mathcal{W}(v) \backslash\{S\}$. To quote the authors, this axiom postulates that the effect (gain or loss) on any player's power of eliminating a single minimal winning coalition from the set of winning ones is the same in any game in which this coalition is minimal winning.

The Shapley-Shubik index Sh is the unique index satisfying the (Null), (Anon) (Trans) and (Eff) axioms ${ }^{1}$ whereas the Banzhaf measure Bz satisfies (Null), (Anon), and (Trans) (or (Lin)). The reader could refer to Lehrer (1988) about the former axiomatization of the Banzhaf value.

A semivalue (Dubey et al., 1981) is any measure of power that satisfies the transfer (Trans), null player (Null) and anonymity (Anon) axioms. Since Sh and $\mathrm{Bz}$ both satisfy these axioms, they are also examples of semivalues.

Alternatives of the Banzhaf index need special mention, especially those that take the swings of voters only in the minimal winning coalition into account. Unfortunately the Deegan-Packel and public good indices do not satisfy the (Trans) axiom, even in their nonnormalized form so they fail to be semivalues.

\section{Möbius transform and dividends}

Harsányi (1963) introduced an alternative, but equivalent representation of TU-games. This approach is based on the observation that the payoff $v(S)$ of a coalition $S$ is composed of 3 parts: (i) the intrinsic value of its members, as singletons, (ii) the added value of cooperation among subsets of these members and finally (iii) the added value of forming $S$ as an improvement over all existing forms of cooperation. When put this way we can see that only item (iii) is a merit of forming $S$, while the rest of the value is generated in subsets of $S$. This item (iii) is referred to as the (Harsányi) dividend of coalition $S$. Turning our formula around, expressing the dividends from the payoffs and extending it to the empty set we obtain the following definition.

\footnotetext{
${ }^{1}$ In the characterisation of the Shapley value for general TU games (Lin) replaces (Trans).
} 
Definition 1 Let $v$ be a TU-game. For each coalition $T$, the dividend $\Delta^{v}(T)$ is defined, recursively, as follows.

$$
\begin{aligned}
\Delta^{v}(\emptyset) & =0 \\
\Delta^{v}(T) & =v(T)-\sum_{S \subsetneq T} \Delta^{v}(S) \text { if } t \geq 1 .
\end{aligned}
$$

Moreover, (4) can be given by the explicit formula

$$
\Delta^{v}(T)=\sum_{S \subseteq T}(-1)^{t-s} v(S), \quad \forall T \in 2^{N} .
$$

For instance, the dividend of a singleton $\{i\}$ in the game $v$ is its worth $v(i)$ itself (this is item (i) above), and the dividend of the coalition $\{i, j\}$ is the difference $v(\{i, j\})-[v(i)+v(j)]$. When $T=\{i, j, k\}$, one might think that $\Delta^{v}(T)$ would be given by $v(T)-[v(i)+v(j)+v(k)]$. However, this expression must already include the dividends of each pair $\{i, j\},\{i, k\}$ and $\{j, k\}$.

Actually, $\Delta^{v}(S)$ measures the pure contribution of cooperation of $T$ (Billot and Thisse, 2005) since one can interpret it as the contribution of cooperation within the coalition $T$ independently of what cooperation brings about in all possible subcoalitions that could have been formed before the coalition $T$ is determined. This representation or the mapping $T \mapsto \Delta^{v}(T)$ is also known as the Möbius transform of the game $v$ (Rota, 1964).

The mapping is bijective and the two forms are equivalent: Given the Möbius transform $\Delta^{v}$ it is possible to retrieve the characteristic function $v$ Indeed, by rewriting (4), we have, for any coalition $T$

$$
\begin{aligned}
v(T) & =\sum_{S \subseteq T} \Delta^{v}(S) \\
& =\sum_{S \in 2^{N}} \Delta^{v}(S) u_{S}(T) .
\end{aligned}
$$

Consequently, any cooperative game, and thus any voting game, can be expressed as a linear combination of unanimity games. The family of unanimity games forms a basis of the vector space of cooperative games with dividends as coordinates.

Now, let $\sum_{S \in 2^{N}} \Delta^{v}(S) u_{S}$ be the (unique) representation of the game $v$ as a linear combination of unanimity games. Calculating the Shapley-Shubik 
or Banzhaf measures of a player using this decomposition is actually quite simple. First observe that

$$
\begin{aligned}
\mathrm{Sh}_{i}\left(u_{S}\right)=\beta_{i}\left(u_{S}\right) & = \begin{cases}\frac{1}{s}, & \text { if } i \in S \\
0, & \text { otherwise }\end{cases} \\
\mathrm{Bz}_{i}\left(u_{S}\right) & = \begin{cases}2^{1-s}, & \text { if } i \in S \\
0, & \text { otherwise. }\end{cases}
\end{aligned}
$$

As the Shapley-Shubik index and the Banzhaf measure are semivalues, by the (Trans) axiom they can be written in terms of dividends:

Proposition 2 (Owen, 1986) For all games $v \in \mathcal{G}_{V}(N)$, and all players $i \in N$

$$
\begin{aligned}
\operatorname{Sh}_{i}(v) & =\sum_{S \subseteq N: i \in S} \frac{\Delta^{v}(S)}{s}, \\
\mathrm{Bz}_{i}(v) & =\sum_{S \subseteq N: i \in S} 2^{1-s} \Delta^{v}(S) .
\end{aligned}
$$

As it is underlined above, the Shapley-Shubik index provides an insight on the interpretation of the dividends: each is equitably shared among the players who belong to the associate coalition. By adding all these contributions for a given player, $\mathrm{Sh}_{i}$ is formed.

\section{Alternative formulae}

Let $v$ be a voting game in $\mathcal{G}_{V}(N)$. Such a game only takes values 0 and 1 , corresponding to losing and winning coalitions. Its dividends, however may take other integer values - how can we interpret the dividends of a voting game? As a first answer, let us consider the Möbius transform of a unanimity game. For any nonempty coalition $S$

$$
\Delta^{u_{S}}(T)=\left\{\begin{array}{l}
1 \text { if } T=S \\
0 \text { otherwise }
\end{array}\right.
$$

Indeed, by applying (6) on the mapping described above, we easily retrieve the game $u_{S}$. This confirms the interpretation of dividends, in the sense that in the unanimity game $u_{S}$ only coalition $S$ has a "weight" in terms of dividends.

Now consider a voting game $v=u_{S}+u_{S^{\prime}}-u_{S \cup S^{\prime}}$ with two minimal winning coalitions $S$ and $S^{\prime}$. 


$$
\Delta^{v}(T)=\left\{\begin{array}{l}
1 \text { if } T=S \text { or } S^{\prime}, \\
-1 \text { if } T=S \cup S^{\prime}, \\
0 \text { otherwise. }
\end{array}\right.
$$

Interestingly, the dividend of coalition $S \cup S^{\prime}$ is the opposite of the ones of $S$ and $S^{\prime}$, which can be explained by the double impact of the two (minimal) winning coalitions on their union. This impact must be balanced by a negative dividend since coalition $S \cup S^{\prime}$ has the same influence on the game as those of $S$ and $S^{\prime}: S \cup S^{\prime}$ is winning but not "more winning" than $S$ and $S^{\prime}$.

As it is true that the dividends of $v$ give insight on the contribution of coalitions of players, that is even more true about the minimal winning coalitions. And as the calculation of the Shapley-Shubik and the Banzhaf indices are based on the set of winning coalitions, it is also possible to compute them with the sole information of the set of minimal winning coalitions. We propose here such formulae.

For any family of coalitions $\mathcal{S}=\left\{S_{1}, \ldots, S_{m}\right\}$, let $\widehat{\mathcal{S}}=\bigcup_{i=1}^{m} S_{i}$.

Proposition 3 Let $v$ be any voting game in $\mathcal{G}_{V}(N)$. Then

$$
v=\sum_{\mathcal{S} \subseteq \mathcal{M}(v): \mathcal{S} \neq \emptyset}(-1)^{|\mathcal{S}|-1} u_{\widehat{\mathcal{S}}}
$$

Proof: Let $T$ be any coalition, and $\mathcal{T}=\{M \in \mathcal{M}(v): M \subseteq T\}$ be the collection of all minimal winning coalitions included in $T$. Note that $\mathcal{T}$ is nonempty if and only if $T \in \mathcal{W}(v)$. Besides, for any subset $\mathcal{S}$ of $\mathcal{M}(v)$, $u_{\widehat{\mathcal{S}}}(T)=1$ if and only if $\widehat{\mathcal{S}} \subseteq T$, that is, $T$ includes every coalition of $\mathcal{S}$. As coalitions in $\mathcal{T}$ are also minimal winning, this is equivalent to having $\mathcal{T}$ contain every element of $\mathcal{S}$, that is, $\mathcal{S} \subseteq \mathcal{T}$. 
Consequently, if $T$ is winning,

$$
\begin{aligned}
\sum_{\mathcal{S} \subseteq \mathcal{M}(v): \mathcal{S} \neq \emptyset}(-1)^{|\mathcal{S}|-1} u_{\widehat{\mathcal{S}}}(T) & =\sum_{\mathcal{S} \subseteq \mathcal{T}: \mathcal{S} \neq \emptyset}(-1)^{|\mathcal{S}|-1} u_{\widehat{\mathcal{S}}}(T) \\
& =\sum_{\mathcal{S} \subseteq \mathcal{T}: \mathcal{S} \neq \emptyset}(-1)^{|\mathcal{S}|-1} \\
& =-\sum_{\mathcal{S} \subseteq \mathcal{T}: \mathcal{S} \neq \emptyset}(-1)^{|\mathcal{S}|} \\
& =-\sum_{\mathcal{S} \subseteq \mathcal{T}}(-1)^{|\mathcal{S}|}+1 \\
& =-\sum_{k=0}^{|\mathcal{T}|} \sum_{\mathcal{S} \subseteq \mathcal{T}:|\mathcal{S}|=k}(-1)^{k}+1 \\
& =-\sum_{k=0}^{|\mathcal{T}|}\left(\begin{array}{c}
|\mathcal{T}| \\
k
\end{array}\right)(-1)^{k}+1 \\
& =-(1-1)^{|\mathcal{T}|}+1=1=v(T),
\end{aligned}
$$

where the first equality of the last line holds since $|\mathcal{T}| \neq 0$. On the other hand, if $T$ is not winning, then $\mathcal{T}$ is empty, and the right member of (13) is still valid but has no term, and thus vanishes, so (12) is again true, which concludes the proof.

The novelty of this expression lies in the fact that only the set of minimal winning coalitions is used when expressing $v$ in terms of unanimity games.

Note that linear combinations of voting games are more meaningful in cooperative game theory, since the sum of two voting games, or a scalar times a voting game are not in general a voting game. However, we showed that such combinations make sense in the Proposition above. In addition, linearity enables to establish the following result:

Lemma $4 \phi: \mathcal{G}_{V}(N) \rightarrow \mathbb{R}^{n}$ is a semivalue if and only if one of the following equivalent conditions holds.

1. $\exists \alpha_{1}, \ldots, \alpha_{n} \in \mathbb{R}$ such that for all $i, \phi_{i}(v)=\sum_{S \subseteq N: i \in S} \alpha_{|S|}(v(S)-v(S \backslash$ $i)$ ).

2. $\exists \beta_{1}, \ldots, \beta_{n} \in \mathbb{R}$ such that for all $i, \phi_{i}(v)=\sum_{S \subseteq N: i \in S} \beta_{|S|} \Delta^{v}(S)$.

3. $\exists \gamma_{1}, \ldots, \gamma_{n} \in \mathbb{R}$ such that for all $i, \phi_{i}(v)=\sum_{\mathcal{S} \subseteq \mathcal{M}(v): i \in \widehat{\mathcal{S}}} \gamma_{|\widehat{\mathcal{S}}|}(-1)^{|\mathcal{S}|-1}$. 
Furthermore

$$
\begin{aligned}
\beta_{j}=\gamma_{j} & =\sum_{k=0}^{n-j}\left(\begin{array}{c}
n-j \\
k
\end{array}\right) \alpha_{j+k}, \quad \forall j=1, \ldots, n \\
\alpha_{j} & =\sum_{k=0}^{n-j}(-1)^{k}\left(\begin{array}{c}
n-j \\
k
\end{array}\right) \beta_{j+k}, \quad \forall j=1, \ldots, n .
\end{aligned}
$$

As a consequence, we establish our main result.

Theorem 5 For any $v \in \mathcal{G}_{V}(N)$, and all $i \in N$,

$$
\begin{aligned}
\operatorname{Sh}_{i}(v) & =\sum_{\mathcal{S} \subseteq \mathcal{M}(v): \mathcal{S} \neq \emptyset} \frac{(-1)^{|\mathcal{S}|-1} \cdot \mathbf{1}_{\widehat{\mathcal{S}}}(i)}{|\widehat{\mathcal{S}}|}, \\
\mathrm{Bz}_{i}(v) & =\sum_{\mathcal{S} \subseteq \mathcal{M}(v): \mathcal{S} \neq \emptyset}(-1)^{|\mathcal{S}|-1} \cdot 2^{1-|\widehat{\mathcal{S}}|} \cdot \mathbf{1}_{\widehat{\mathcal{S}}}(i),
\end{aligned}
$$

where $\mathbf{1}_{A}$ denotes the indicator function of the set $A$.

Proof: The result straightforwardly follows from (7), (8) and linearity of the power indices Sh and Bz.

Unfortunately, this representation does not extend to other indices, such as the public good-, Deegan-Packel, Johnston, or Colomer-Martinez indices due to their "nonlinearity", even in nonnormalized forms.

In the formulae above, the coalitions under the form $\widehat{\mathcal{S}}$ may appear more than once. It is possible to avoid this thanks to the Möbius transform. By identification of the linear coefficient of $u_{S}$ in (12) and (6), we get:

$$
\Delta^{v}(T)=\sum_{\mathcal{S} \subseteq \mathcal{M}(v): \widehat{\mathcal{S}}=T}(-1)^{|\mathcal{S}|-1} .
$$

Let $\overline{\mathcal{M}}$ be the closure of $\mathcal{M}(v)$ with respect to the union operation. This set can be constructed by looking at all subsets of $\mathcal{M}(v)$ and taking each union. Formally $\overline{\mathcal{M}}=\{\widehat{\mathcal{S}}: \mathcal{S} \subseteq \mathcal{M}(v), \mathcal{S} \neq \emptyset\}$. Note that $\mathcal{M}(v) \subseteq \overline{\mathcal{M}}(v) \subseteq \mathcal{W}(v)$, with strict inclusion possible on both sides. Then, for any voting game:

$$
v=\sum_{T \in \overline{\mathcal{M}}(v)} \Delta^{v}(T) u_{T}
$$

This yet another reformulation of the game suggests an even simpler calculation of our measures. Similar formulas can be found for other semivalues. 
Corollary 6 For any $v \in \mathcal{G}_{V}(N)$, and all $i \in N$,

$$
\begin{aligned}
\operatorname{Sh}_{i}(v) & =\sum_{T \in \overline{\mathcal{M}}(v): i \in T} \frac{\Delta^{v}(T)}{t}, \\
\mathrm{Bz}_{i}(v) & =\sum_{T \in \overline{\mathcal{M}}(v): i \in T} \Delta^{v}(T) \cdot 2^{1-t} .
\end{aligned}
$$

\section{Applications}

With $\overline{\mathcal{M}}(v) \subsetneq \mathcal{W}(v)$ possible our approach can, in some cases, simplify the calculations drastically. In this section we show some examples that illustrate this point.

In games where set of voters is large, but $\mathcal{M}(v)$ is small it is clearly preferable to use a calculation that relies on the latter. In that case, even good algorithms derived from classical formulae will take much longer.

Example 1 Let $N$ be a large assembly of voters, partitioned into $\{1,2,3,4\}$ and the rest $N^{\prime}$ of voters. We consider the game $v$ with the following rules that are necessary to pass a bill:

1. Voter 1 has a veto, that is, the presence of 1 is necessary for a coalition to be winning.

2. The presence of 2 and 3 is also sufficient (in addition to 1 's).

3. If 2 is absent, all members of $N^{\prime}$ must be present, jointly with 3 or 4.

One can easily derive the minimal winning coalitions: $S=\{1,2,3\}, T=$ $N^{\prime} \cup\{1,3\}$, and $U=N^{\prime} \cup\{1,4\}$. Then by applying (16) in Theorem 5, we get, for any $i \in N$

$$
\begin{aligned}
\operatorname{Sh}_{i}(v)= & (-1)^{0}\left(\frac{\mathbf{1}_{S}(i)}{s}+\frac{\mathbf{1}_{T}(i)}{t}+\frac{\mathbf{1}_{U}(i)}{u}\right) \\
& +(-1)^{1}\left(\frac{\mathbf{1}_{S \cup T}(i)}{|S \cup T|}+\frac{\mathbf{1}_{S \cup U}(i)}{|S \cup U|}+\frac{\mathbf{1}_{T \cup U}(i)}{|T \cup U|}\right)+(-1)^{2} \frac{\mathbf{1}_{S \cup T \cup U}(i)}{|S \cup T \cup U|}
\end{aligned}
$$

Thus, we straightforwardly get

$$
\begin{array}{r}
\operatorname{Sh}(v)=\left(\frac{1}{3}+\frac{2}{n-2}-\frac{2}{n-1}, \frac{1}{3}-\frac{1}{n-1}, \frac{1}{3}+\frac{1}{n-2}-\frac{2}{n-1}, \frac{1}{n-2}-\frac{1}{n-1},\right. \\
\left.\frac{2}{n-2}-\frac{2}{n-1}, \ldots, \frac{2}{n-2}-\frac{2}{n-1}\right),
\end{array}
$$


that has the following asymptotic behavior (for large $n$ 's):

$$
\operatorname{Sh}(v)=\left(\frac{1}{3}+\frac{2}{n^{2}}, \frac{1}{3}-\frac{1}{n}, \frac{1}{3}-\frac{1}{n}, \frac{1}{n^{2}}, \frac{2}{n^{2}}, \ldots, \frac{2}{n^{2}}\right) .
$$

In the same way, we also get:

$$
\mathrm{Bz}(v)=\left(\frac{1}{4}+2^{3-n}, \frac{1}{4}-2^{2-n}, \frac{1}{4}, 2^{2-n}, 2^{3-n}, \ldots, 2^{3-n}\right) .
$$

Note that for a large $n$ the set of (non-minimal) winning coalitions is enormous, already defining the underlying simple voting game can be a daunting task.

Note that small cardinalities of $\mathcal{M}(v)$ also occur for weighted games with a large threshold. Having said that, the set $\mathcal{M}(v)$ can be large enough so that considering all possible unions of elements of $\mathcal{M}(v)$ could take very long. However, it is quite common that for a given weighted voting game, the set $\left\{w_{i}: i \in N\right\}$ of all possible different weights is quite small, even if the number of players is great. We show in the following example that the set of possible unions can be reduced to a bit larger set of combinations to explore.

Example 2 Let us consider a council with eight representative parties and a voting situation that can be described as a weighted voting game

$$
v=[10 ; 4,4,2,1,1,1,1,1] .
$$

In this game there are exactly 21 minimal winning coalitions, which induces a sum over $2^{21}$ (more than two million) terms in (16) and (17). However, by identifying the coalitions in which every weight (4, 2 and 1) appears the same number of times, we only have to consider 11 kinds of such coalitions: $\{4,4,2\},\{4,4,1,1\},\{4,2,1,1,1,1\},\{4,4,2,1,1\},\{4,4,2,1,1,1,1\},\{4,4,1,1,1\}$, $\{4,4,1,1,1,1\},\{4,4,2,1,1,1,1,1\},\{4,2,1,1,1,1,1\},\{4,4,2,1,1,1\}$ and $\{4,4,1,1,1,1,1\}$. By using some basic combinatorics, we can use (20) and (21) to get:

$$
\begin{aligned}
\operatorname{Sh}(v) & =\left(\frac{2}{7}, \frac{2}{7}, \frac{5}{42}, \frac{13}{210}, \frac{13}{210}, \frac{13}{210}, \frac{13}{210}, \frac{13}{210}\right), \\
\operatorname{Bz}(v) & =\left(\frac{29}{64}, \frac{29}{64}, \frac{9}{64}, \frac{3}{32}, \frac{3}{32}, \frac{3}{32}, \frac{3}{32}, \frac{3}{32}\right) .
\end{aligned}
$$

Note that such weighted voting games can naturally occur in, for instance, corporations with publicly traded shares. 


\section{Discussion}

Ever since the power measures have been around theorists have been looking for efficient ways of expressing them.

Ours is not the only approach to express power measures in terms of winning coalitions. Kirsch and Langner (2009) also emphasise the importance of building a power index this way. However, their expressions are not quite as straightforward as ours, and the way they have been obtained is much longer, making use of much complicated combinatorics. Introducing the Möbius transform in our approach does not only stress the important link with the set of minimal winning coalitions, but also simplifies the connection between these issues.

Our contribution is to formalise the logical link between power indices and minimal winning coalitions. Our formulae are not only elegant from a logical point of view, but offer an alternative way to calculate power indices and semivalues. This alternative way is not necessarily more efficient over the whole class of simple voting games, but it is much quicker in games with relatively few winning coalitions.

\section{References}

Banzhaf JF (1965) Weighted voting doesn't work: A mathematical analysis. Rutgers Law Review 19:317-343

Billot A, Thisse JF (2005) How to share when context matters: The Möbius value as a generalized solution for cooperative games. Journal of Mathematical Economics 41(8):1007-1029

Deegan J, Packel EW (1978) A New Index of Power for Simple \$n\$-Person Games. International Journal of Game Theory 7(2):113-123

Dubey P (1975) On the uniqueness of the Shapley value. International Journal of Game Theory 4(3):131-139

Dubey P, Weber RJ, Neyman A (1981) Value theory without efficiency. Mathematics of Operations Research 6(1):122-128

Grabisch M, Marichal JL, Roubens M (2000) Equivalent Representations of Set Functions. Mathematics of Operations Research 25(2):157-178

Harsányi JC (1963) A Simplified Bargaining Model of the n-person Cooperative Game. International Economic Review 4(2):194-220 
Holler MJ, Packel EW (1983) Power, Luck and the Right Index. Journal of Economics 43(1):21-29

Kirsch W, Langner J (2009) Power indices and minimal winning coalitions. Social Choice and Welfare 34(1):33-46

Kóczy LA (2011) Beyond Lisbon: Demographic trends and voting power in the European Union Council of Ministers. Mathematical Social Sciences

Laruelle A, Valenciano F (2001) Shapley-Shubik and Banzhaf Indices Revisited. Mathematics of Operations Research 26(1):89-104

Lehrer E (1988) An Axiomatization of the Banzhaf Value. International Journal of Game Theory 17(2):89-99

Owen G (1986) Values of Graph-Restricted Games. SIAM Journal on Algebraic and Discrete Methods 7(2):210

Penrose LS (1946) The elementary statistics of majority voting. Journal of the Royal Statistical Society 109(1):53-57

Rota GC (1964) On the foundations of combinatorial theory I. Theory of Möbius Functions. Zeitschrift für Wahrscheinlichkeitstheorie und Verwandte Gebiete 2(4):340-368

Shapley LS (1953) A Value for n-Person Games. In: Kuhn HW, Tucker A (eds.) Contributions to the theory of games, Princeton University Press pp. 307-317

Shapley LS, Shubik M (1954) A method for evaluating the distribution of power in a committee system. The American Political Science Review 48(3):787-792 